\title{
Genotype-guided antiretroviral regimens in children with multidrug-resistant HIV-1 infection
}

\author{
Gloria Huerta-García', José G. Vazquez-Rosales', José A. Mata-Marín², Leoncio Peregrino-Bejarano', Eric Flores-Ruiz and \\ Fortino Solórzano-Santos ${ }^{3}$
}

BACKGROUND: Genotyping tests were developed to attenuate the impact of viral resistance. Information about the efficacy in genotype base antiretroviral therapy in children is rare and even more in low- and middle-income countries.

METHODS: Sixteen children with antiretroviral therapy (ART) failure and triple-class drug-resistant viruses were included in this study. Protease and retrotranscriptase genotypes were available for all patients. Switch of ART regimen was guided by genotyping data. The primary end point was virological suppression (<50 copies/ml) and immunological improvement after 48 wk of treatment with the new ART regimen.

RESULTS: The median age of the patients was 14.5 y (interquartile range (IQR) 11-16.5). Median HIV-1 RNA viral load was $4.2 \log _{10}$ (IQR: 3.4-4.8). The primary end point was found in 11 children (69\%), and 13 children (81\%) had an HIV-1 RNA viral load <200 copies/ml. Median (IQR) for the baseline CD4+ cell

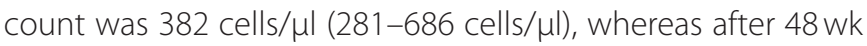
of treatment with the new ART regimen, it was 640 cells/ $\mu$ l (361-936 cells/ $\mu \mathrm{l})(P<0.001)$.

CONCLUSION: Darunavir/ritonavir, raltegravir, and etravirine were well tolerated in the present pediatric population. These drugs provide good options for children exposed to extensive ART. Regimens guided by genotyping data were effective for children who had ART failure and multidrug-resistant HIV-1 infection.

nfants and children who were infected perinatally are now surviving to adulthood with lifelong HIV infection and longterm exposure to combined antiretroviral therapy (ART) (1). Data from the United Kingdom Collaborative HIV Pediatric Study cohort demonstrated that over one-third of children had experienced virological treatment failure and were on a second or subsequent line of therapy at the time of their transition to adult care (2). In the pediatric setting, virological treatment failure occurs most frequently because of poor adherence to ART, and whenever viral replication is inefficiently controlled, virological treatment failure occurs more quickly, allowing the selection of HIV-1 quasispecies resistant to antiretroviral (ARV) drugs (3).
Many innate and external factors have been associated with HIV drug resistance mutations in children, such as being infected perinatally, and extremely high viral load during the first steps of the infection, which can take longer to suppress than that in older children or adults, even when they are on fully active ART regimens (4).

Interpretation of resistance tests in this context can be complex, particularly in the presence of expanding options for ARV drugs, and treatment decisions should be made with the support of pharmacists and clinicians who have expertise in the field (5).

Currently, more than 20 different ARV drugs with six different classes of action have been approved for use in children or adolescents with HIV infection worldwide; most of them are used in Mexico (6).

A good level of adherence is particularly difficult during adolescence, especially with a complex regimen if a once-daily low pill burden option is not available because of prior acquisition of resistance mutations (7).

Ritonavir-boosted darunavir (DRV/r) and raltegravir (RAL) represent potential choices for salvage ART in children and adolescents who have failed to respond to protease inhibitors (PIs) with high-grade PI resistance. Studies of children and adolescents with experience of ART have shown safety and tolerability of $\mathrm{DRV} / \mathrm{r}$ in these younger populations $(8,9)$. DRV/r has been widely recommended as a salvage therapy for children older than $3 \mathrm{y}$ and adolescents with high levels of treatment experience and ART resistance (9).

The aim of the study was to assess the virological and immunological response to an individualized ARV regimen guided by genotyping data in HIV-1-infected children and adolescents with ART experience and resistance. Secondary metabolic effects of the regimen are described.

\section{RESULTS}

Sixteen patients were included. Their median age was $14.5 \mathrm{y}$ (interquartile range (IQR): 11.2-16.7), and 10 were boys. Class B of the CDC classification system of AIDS was found in seven patients (44\%), class C in $6(37 \%)$, and class $\mathrm{A}$ in 3

'Pediatric Infectious Disease Department, Hospital de Pediatría, Centro Médico Nacional "Siglo XXI", IMSS, Ciudad de Mexico, México; ${ }^{2}$ Infectious Diseases Department, Hospital de Infectología, National Medical Center "La Raza”, IMSS, Ciudad de Mexico, México; ${ }^{3}$ Unidad Médica de Alta Especialidad, Hospital de Pediatría, National Medical Center "Siglo XXI", IMSS, Ciudad de Mexico, México. Correspondence: Gloria Huerta-García (gloriahuerta@gmail.com)

Received 12 October 2015; accepted 28 December 2015; advance online publication 13 April 2016. doi:10.1038/pr.2016.53 
Table 1. Baseline characteristics $(N=16)$

\begin{tabular}{|c|c|c|c|c|}
\hline & \multicolumn{3}{|c|}{ ART regimen } & \multirow[b]{2}{*}{$\begin{array}{l}\text { All patients } \\
\quad(n=16)\end{array}$} \\
\hline & $\begin{array}{c}\mathrm{DRV} / \mathrm{r}+\mathrm{OBR} \\
(n=13)\end{array}$ & $\begin{array}{c}\mathrm{TPV} / \mathrm{r}+\mathrm{OBR} \\
(n=2)\end{array}$ & $\begin{array}{c}\text { Without PI (RAL, ETV, } \\
\text { TDF) }(n=1)\end{array}$ & \\
\hline & \multicolumn{4}{|c|}{ Median (IQR) } \\
\hline Duration of virological failure, years & $7(4-10)$ & $5.5(3-8)$ & 10 & $7.5(3.2-10)$ \\
\hline \multicolumn{5}{|l|}{ CDC class, $n(\%)$} \\
\hline A & $3(24 \%)$ & 0 & 0 & $3(18.7 \%)$ \\
\hline B & $5(38 \%)$ & $2(100 \%)$ & 0 & $7(43.7 \%)$ \\
\hline$C$ & $5(38 \%)$ & 0 & $1(100 \%)$ & $6(37.5 \%)$ \\
\hline
\end{tabular}

ART, antiretroviral therapy; DRV/r, darunavir; IQR, interquartile range; OBR, optimized background regimen; Pl, protease inhibitor; RAL, raltegravir.

Table 2. History of antiretroviral treatment

\begin{tabular}{|c|c|c|c|c|}
\hline & \multicolumn{3}{|c|}{ ART regimen } & \multirow[b]{2}{*}{$\begin{array}{l}\text { All patients } \\
\quad(n=16)\end{array}$} \\
\hline & $\begin{array}{c}\mathrm{DRV} / \mathrm{r}+\mathrm{OBR} \\
(n=13)\end{array}$ & $\begin{array}{c}\mathrm{TPV} / \mathrm{r}+\mathrm{OBR} \\
(n=2)\end{array}$ & $\begin{array}{l}\text { Without PI (RAL, } \\
\text { ETV, TDF) }(n=1)\end{array}$ & \\
\hline \multicolumn{5}{|l|}{ Previous ARV treatment } \\
\hline$\geq 1 \mathrm{Pl}, n(\%)$ & $13(100 \%)$ & $2(100 \%)$ & $1(100 \%)$ & $16(100 \%)$ \\
\hline \multicolumn{5}{|l|}{ Baseline resistance } \\
\hline \multicolumn{5}{|l|}{ Primary IAS_USA mutations ${ }^{10}$} \\
\hline PI RAMs & $4(1-5)$ & $5(3-6)$ & 6 & $4(1-6)$ \\
\hline NRTI RAMs & $4(0-6)$ & $3(2-4)$ & 4 & $4(0-6)$ \\
\hline
\end{tabular}

ART, antiretroviral therapy; ARV, antiretroviral; DRV/r, darunavir; IAS-USA, International antiviral society—USA; IQR, interquartile range; NNRTI, non-nucleoside reverse-transcriptase inhibitors; NRTI, nucleoside reverse-transcriptase inhibitors; OBR, optimized background regimen; PI, protease inhibitor; RAL, raltegravir; RAMs, resistance-associated mutations.

(19\%), and the median number of previous ART regimens was 5 (IQR: 4-6) (Tables 1 and 2). None of the patients included had opportunistic or other infections during the study.

When we analyzed the optimized background regimen according to the Stanford HIV database (HIVdb), we did not find any difference between the genotype sensitivity score (GSS) of optimized background regimen in those patients with virological treatment failure and those with HIV-1 RNA $<50$ copies $/ \mathrm{ml}$.

The median calculated GSS of the current regimen was 2.12 (IQR: 1.75-2.5). Twelve (75\%) patients had a GSS $\geq 2$ taking in to account all components included in prescribed regimen, or only two patients (13\%) if we measure the GSS excluding a completely active component (RAL or DRV). There was no association between GSS and a greater possibility of treatment failure ( $P=0.87$; Table 3$)$.

The median of baseline HIV-1 RNA was $4.20 \log _{10}$ (IQR: $3.47-4.88 \log _{10}$ ). After $48 \mathrm{wk}$ of treatment, $69 \%$ had $<50$ copies/ $\mathrm{ml}$, median $1.33 \log _{10}$ (IQR: $1.30-2.26 \log _{10}$ ), and $81 \%$ of the patients $(n=13)$ had an HIV-1 RNA $<200$ copies/ml; $P<0.001$ compared with baseline.

For five patients with virological treatment failure, one had virological rebound, and four never achieved an HIV-1 RNA load of $<50$ copies/ml $\left(1.69 \log _{10}\right)$; two of these four had $<200$ copies $/ \mathrm{ml}$.

The median (IQR) for the baseline $\mathrm{CD} 4^{+}$cell count was 382 cells $/ \mu \mathrm{l}$ (281-686 cells/ $\mu \mathrm{l})$, whereas after $48 \mathrm{wk}$ of treatment, the value was $640 \mathrm{cells} / \mu \mathrm{l}$ (361-936 cells/ $\mu \mathrm{l})$. This increase was statistically significant $(P<0.001)$.

Fasting lipid profiles (total cholesterol and triglycerides) were not significantly different from basal levels after $48 \mathrm{wk}$ of treatment. Hypercholesterolemia and hypertriglyceridemia were found in 12 and $20 \%$ of patients at baseline and in 25 and $50 \%$ after $48 \mathrm{wk}$ of treatment $(P=0.50,0.18)$, respectively.

There was no significant increase in creatinine or glucose compared with baseline values (Table 4). 
Table 3. ART characteristics, optimized background regimen and outcomes

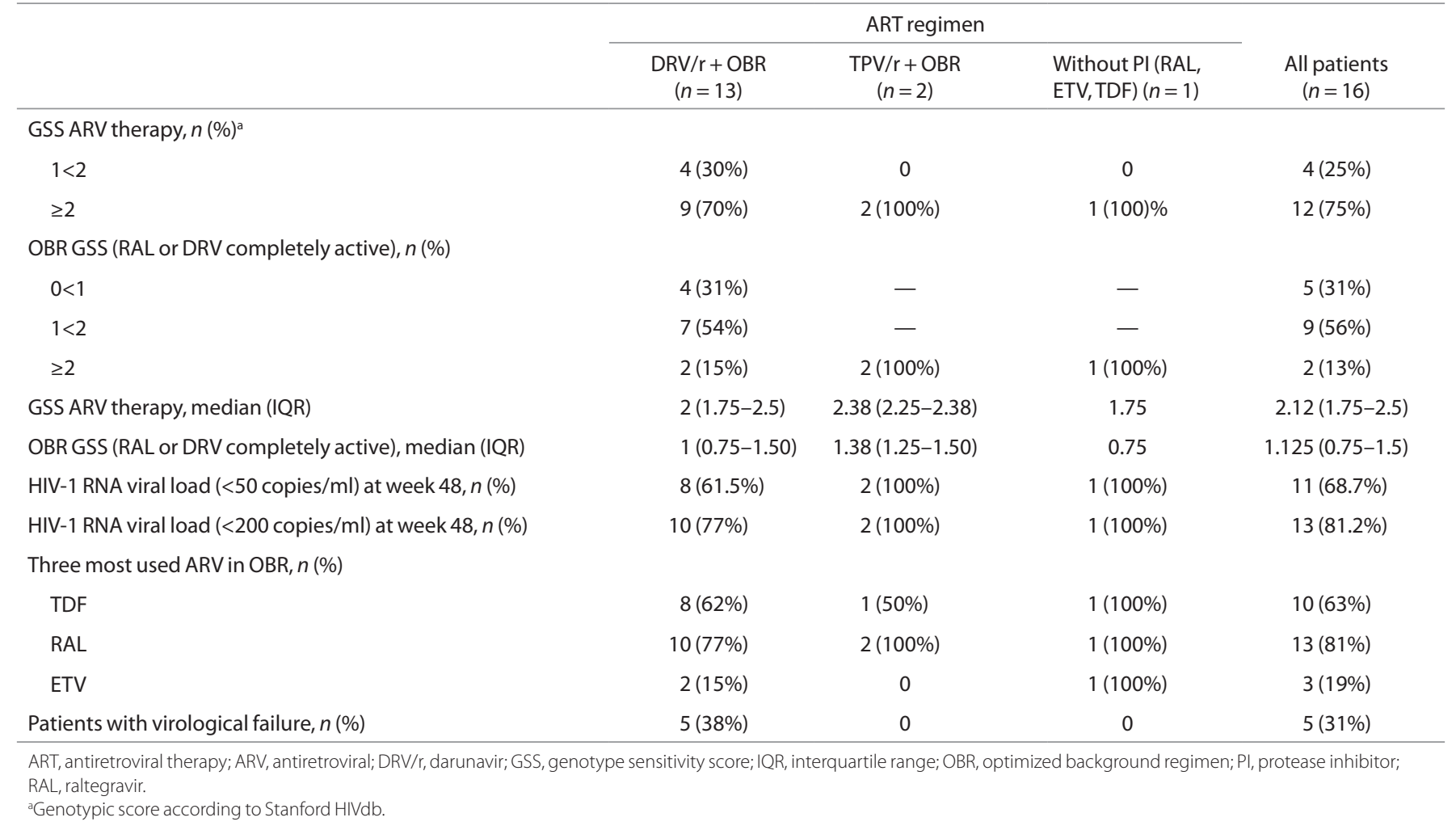

Median weight-for-age and height-for-age ( $z$-scores) were stable over the period of the study; all patients obtained or maintained WHO clinical treatment stage I (Table 4).

The most common drug resistance mutations present for nucleoside reverse-transcriptase inhibitors were M184V (81\%), M41L (56\%), T215Y/I/F (50\%), K219Q/E (37\%), and K70R (31\%), for non-nucleoside reverse-transcriptase inhibitors were K103N (31\%), Y188L (25\%), and G190S/A (25\%), and for PIs were L54V (100\%), V82A/S (75\%), M46I/L (69\%), and L90M (44\%) (Table 5).

We were not able to evaluate adverse events fully because of the retrospective study design. However, the regimen was not suspended because of side effects in any of the children.

\section{DISCUSSION}

We showed the feasibility of achieving a virological and immunological response with an antiretroviral regimen guided by genotype in this cohort of Mexican children and adolescents infected with HIV perinatally, even after the selection of triple-class drug resistance mutations. All patients who achieved viral suppression showed improved or stable $\mathrm{CD} 4^{+}$cell counts, even after long-standing treatment failure. At the end of this study, all of the patients in this report were classified as having a WHO class 1 HIV infection, and they did not present opportunistic infections during the study; however, the prescription of an effective combination therapy for HIV-infected children harboring an MDR virus is one of the greatest challenges in the clinical management of children with HIV infection $(11,12)$. In this study, the basal sample had a susceptibility to DRV,
Tipranavir (TPV), Etravirine (ETV), and Tenofovir (TDF) of $87,37,87$, and 50\%, respectively, according to the Stanford HIVdb; in addition, RAL was considered to be fully active.

Routine clinical use of DRV/r and/or RAL in children in hospitals with or without economic resources is limited: a cohort in Botswana of four TC-MDR pediatric patients on $\mathrm{DRV} / \mathrm{r}$ and/or RAL-based regimens all achieved viral suppression showed improved/stable $\mathrm{CD} 4^{+}$cells counts and obtained or maintained WHO clinical treatment stage I, even after longstanding virological/immunological failure (11). We are not aware of previously published reports from Latin America or Mexico. Our data are similar to those from a French cohort where 11/12 adolescents with extensive experience of ARV treatment achieved viral suppression $(<400$ copies $/ \mathrm{ml})$ with a regimen of DRV/r plus RAL plus ETV (12). Despite ARV resistance, the susceptibility to some drugs, such as new PIs (TPV and DRV) and non-nucleoside reverse-transcriptase inhibitors (ETV and rilpivirine), was maintained in a cohort of Spanish children and adolescents with perinatally acquired HIV infections and TC-MDR (13).

Some studies show the efficacy of some ARV drugs used separately in children, such as the phase 2 PIANO study that shows the efficacy of ETV treatment in HIV-1-infected children and adolescents who previously used EFV or NVP; $56 \%$ of patients ( $68 \%$ children; $48 \%$ adolescents) achieved virological response (14). In another trial, RAL was evaluated as part of a salvage treatment in a multicenter retrospective study of 19 MDR children and adolescents. After receiving RAL-based therapy, 4/19 (21\%) patients achieved an HIV-1 RNA load of 
Table 4. End points after 48 wk of treatment

\begin{tabular}{|c|c|c|c|}
\hline \multirow[b]{2}{*}{ Outcomes } & \multicolumn{2}{|c|}{ Median (IQR) } & \multirow[b]{2}{*}{$P$} \\
\hline & Baseline & Week 48 & \\
\hline $\begin{array}{l}\text { CD4 }{ }^{+} \text {cells count, } \\
\text { (cells/ } / \text { l) }\end{array}$ & $382(281-687)$ & $640(361-936)$ & 0.001 \\
\hline $\begin{array}{l}\text { HIV-1 RNA viral } \\
\text { load }\left(\log _{10}\right)\end{array}$ & $4.20(3.47-4.88)$ & $1.33(1.30-2.26)$ & $<0.0001$ \\
\hline Weight, $Z$ score & $-1.66(-2.24,-0.80)$ & $-1.46(-2.05,-0.81)$ & 0.932 \\
\hline Height, $Z$ score & $-2.05(-2.87,-1.13)$ & $-1.75(-2.72,-1.41)$ & 0.187 \\
\hline BMI, Z score & $-0.36(-1.56,0.20)$ & $-0.3(-2.04,0.07)$ & 0.650 \\
\hline Cholesterol (mg/dl) & $145(137-171)$ & $165(133-204)$ & 0.196 \\
\hline Triglycerides (mg/dl) & $118(86-143)$ & 149 (79-191) & 0.112 \\
\hline Creatinine (mg/dl) & $0.5(0.42-0.67)$ & $0.48(0.39-0.57)$ & 0.141 \\
\hline Glucose (mg/dl) & $83(75-91)$ & $85.8(79-90.3)$ & 0.382 \\
\hline
\end{tabular}

$<400$ copies, and 13/17 (68\%) reached an HIV-1 RNA load of $<50$ copies; $\mathrm{CD}^{+}$cell count recovery was observed in 17/19 (89\%) patients. No deaths, AIDS-defining illnesses, or symptoms of severe intolerance were recorded in this multicenter cohort (15).

In this study, a mild increase in lipids was found, but this was not statistically significant, maybe because of the previous use of other PIs having a worse effect on lipids. The increase in lipids was higher in teens than in children (58 vs. $12 \%$ ), and it was probably associated with longer exposure to the ART, hormonal changes, and more flexible diets, as described in other reports (16).

Children infected perinatally are usually exposed to many ART regimens, and once in adolescence and young adulthood, they have typically accumulated drug-resistant viruses that can be significantly difficult to control (17). Infants have a higher risk of developing resistance compared with adolescents and adults because younger children have higher viral loads, and during treatment, their family members cannot provide accurate dosing, because of the limitations in formulations of antiretroviral drugs, which can lead to subtherapeutic drug concentrations (17).

There are a considerable number of children and adolescents who acquired their HIV-infection in the pre-HAART (highly active antiretroviral therapy) era. Fifty percent of patients in the present cohort (eight patients) had received a large number of antiretroviral regimens, with a median of five regimens in the entire cohort; consequently, drug resistance mutations complicate selection of new ART regimens (18).

There are now six therapeutic classes of medications available, although not all are licensed for use in children, and some of them are not available in Mexico (e.g., dolutegravir, cobicistat). Most ARV (developed in tablet form) are impractical for children aged less than $5 \mathrm{y}$, and other options, such as special liquid formulations, small pills, or chewable formulations, are not all available yet, and if they do exist, they are not available in Mexico. Although older children can take tablets, adult doses are often too large (19). The main reason for multiple
Table 5. Major drug-associated mutations

\begin{tabular}{|c|c|}
\hline Mutation & $n(\%)$ \\
\hline \multicolumn{2}{|c|}{ Associated with NRTIs } \\
\hline L184V & $13(81 \%)$ \\
\hline M41L & $9(56 \%)$ \\
\hline $\mathrm{T} 215 \mathrm{Y} / \mathrm{I} / \mathrm{F}$ & $8(50 \%)$ \\
\hline K219Q/E & $6(37 \%)$ \\
\hline D67N & $5(31 \%)$ \\
\hline K70R & $5(31 \%)$ \\
\hline T69N & $3(19 \%)$ \\
\hline L74V & $1(6 \%)$ \\
\hline V75M & $1(6 \%)$ \\
\hline \multicolumn{2}{|c|}{ Associated with NNRTIs } \\
\hline $\mathrm{K} 103 \mathrm{~N} / \mathrm{S}$ & $5(31 \%)$ \\
\hline G190A/C/S/V & $4(25 \%)$ \\
\hline $\mathrm{Y} 188 \mathrm{C} / \mathrm{L}$ & $4(25 \%)$ \\
\hline K101E/P & $2(12 \%)$ \\
\hline Y181C & $1(6 \%)$ \\
\hline \multicolumn{2}{|c|}{ Associated with Pls } \\
\hline $\mathrm{L} 54 \mathrm{~L} / \mathrm{V}$ & $16(100 \%)$ \\
\hline $\mathrm{V} 82 \mathrm{~A} / \mathrm{S}$ & $12(75 \%)$ \\
\hline M46I/L & $11(69 \%)$ \\
\hline L90M & $7(44 \%)$ \\
\hline $184 \mathrm{~V}$ & $6(37 \%)$ \\
\hline $\mathrm{T} 74 \mathrm{~A} / \mathrm{P} / \mathrm{S}$ & $3(19 \%)$ \\
\hline L76V & $3(19 \%)$ \\
\hline G48V & $2(12 \%)$ \\
\hline L50V & $2(12 \%)$ \\
\hline Q58E & $2(12 \%)$ \\
\hline V32I & $1(6 \%)$ \\
\hline
\end{tabular}

NNRTI, non-nucleoside reverse-transcriptase inhibitors; NRTI, nucleoside reverse-transcriptase inhibitors; Pl, protease inhibitor.

regimens before genotyping for salvage therapy in this population was the lack of a pediatric indication for new ARV drugs recently accepted for pediatric use.

Although our experience reported here involves a small number of patients, sustained virological suppression has been achieved in patients who previously experienced persistent viremia with complete adherence to current ART; immunological indices improved or they were stable, and no intolerance or toxicity was noted in any patient, including when high levels of resistance to PIs were present. No deaths or AIDSdefining clinical conditions were observed in our cohort during the salvage regimen.

Genotypic resistance testing following virological treatment failure in adults has been shown to lead to modest virological benefits $(20,21)$. PENTA 8 was a randomized trial of genotypic resistance testing vs. clinician-guided switch in children with failing virological treatment, and this study showed no 
virological or immunological benefit at $96 \mathrm{wk}$ (22); however, this finding should be interpreted with caution, because second-generation PIs and TDF were not available at the time of the study. In the present series, a favorable response observed in 13 of 16 patients who received combination therapy with the salvage drugs could be attributed to an individualized analysis of genotype by an expert committee and recommendation of the individualized ARV regimen for each patient.

The present study has some limitations; these include the small sample size, retrospective method, short follow-up time, and possible selection bias because of the type of the hospitals, which are centers of reference.

This population is increasing within the time, and the analysis continues.

In conclusion, there are specific challenges in treating HIVinfected children, including uncertainty about the best time to start treatment, the need for more pediatric formulations, the lack of pharmacokinetic studies for new drugs in children, and incomplete dosing guidelines for children. The genotypic resistance testing is a valuable tool to conform an effective antiretroviral regimen in highly experimented children.

\section{METHODS}

\section{Design}

A retrospective multicenter study of a cohort of HIV-1-infected children with ART experience was conducted. The first end point analyzed was an HIV-1 RNA viral load of $<50$ copies $/ \mathrm{ml}$ after the patients had completed $48 \mathrm{wk}$ of treatment. The secondary end point was an HIV-1 RNA viral load of $<200$ copies $/ \mathrm{ml}$ and any increase in the $\mathrm{CD} 4^{+}$ cell count.

The Institutional Review Board and Ethics Local Committee in the Hospital de Pediatría CMN SXXI reviewed and approved this study, with reference number: R-2012-3603-81.

\section{Patients}

Children aged $<18$ y with an HIV-1 infection acquired perinatally and ART failure were included. Patients were previously treated with at least three classes of ARV drugs including nucleoside analog reversetranscriptase inhibitors, non-nucleoside reverse-transcriptase inhibitors, and PIs, with documented triple-class drug resistance mutations. Due to the retrospective nature of the study, written informed consent was not necessary.

An individualized ART was designed for each patient. The regimen included three to four ARV agents, with two or more fully active drugs according to HIV-1 resistance testing and previous ARV drug experience.

\section{Measurements}

Clinical history regarding ARV regimens, $\mathrm{CD}^{+}$cell counts, HIV-1 RNA viral load, and serum laboratory parameters at the beginning of the therapy and at 24 and 48 wk later were recorded. After genotyping, and considering previous use of ARV regimens, an expert committee evaluated each case to determine the best option for a salvage regimen.

Mutations were assessed from plasma HIV-1 pol sequences using the Stanford HIVdb. The presence of resistance was defined according to Stanford HIVdb (SS) ranges as follows: 0-9, susceptible; 10-14, potential low-level resistance; 15-29, low-level resistance; 30-59, intermediate resistance; and $\geq 60$, high-level resistance.

The GSS is based on the total number of drugs in an ARV regimen to which the HIV isolate has genotypic sensitivity, as deduced from gene sequence and mutation analyses. This was calculated based on the drug resistance scores extracted from the Stanford HIVdb. Each ARV drug was assigned a score according to the five-level Stanford HIVdb interpretation: 1.00 (susceptible), 0.75 (potential low-level resistance), 0.50 (low-level resistance), 0.25 (intermediate resistance), and 0.00 (high-level resistance). The GSS is defined as the sum of all scores for all the drugs in the regimen. The arithmetic sum of the individual score for the specific drugs provided the total GSS for that treatment. We classified the total GSS score into the following categories: $<1,1$ to 2 , and $\geq 2$. The 0 to 1 group contains viral sequences almost entirely resistant to the drugs in their regimen, and the $\geq 2$ group contains viral sequences susceptible to two or more drugs given in their ARV regimen.

The effectiveness of ART was evaluated using the percentages of undetectable HIV-1 RNA viral load after 48 wk of treatment. Changes in $\mathrm{CD} 4^{+}$cell counts were also evaluated.

Metabolic end points were changes in fasting lipid levels (total cholesterol and triglycerides), in glucose, and in creatinine from baseline and at $48 \mathrm{wk}$. Including weight, height, and BMI Z scores before and at final analysis.

\section{Statistical Analyses}

Baseline characteristics are summarized using medians and IQR for continuous variables and proportions for categorical variables.

The differences in the CD $4^{+}$cell count, HIV-1 RNA viral load, and lipids, at baseline and after $48 \mathrm{wk}$ of treatment were calculated. Because the data followed an irregular distribution, they were compared using a Wilcoxon signed-rank test. A Mann-Whitney $U$-test was used to evaluate differences between ARV GSS scores, and viral and immunological end points.

All analyses were conducted using SPSS software (IBM SPSS for Windows, version 19.0, 2010 release, IBM, Armonk, NY).

\section{STATEMENT OF FINANCIAL SUPPORT}

No financial assistance was received to support this study.

Disclosure: There are no disclosures.

\section{REFERENCES}

1. Sohn AH, Hazra R. The changing epidemiology of the global paediatric HIV epidemic: keeping track of perinatally HIV-infected adolescents. J Int AIDS Soc 2013;16:18555.

2. Foster C, Judd A, Tookey P, et al.; Collaborative HIV Paediatric Study (CHIPS). Young people in the United Kingdom and Ireland with perinatally acquired HIV: the pediatric legacy for adult services. AIDS Patient Care STDS 2009;23:159-66.

3. Larru B, de Mendoza C, Bellón JM, et al. Predictive factors of virological success to salvage regimens containing protease inhibitors in HIV-1 infected children. BMC Infect Dis 2007;7:55.

4. Le Doare K, Mackie NE, Kaye S, Bamford A, Walters S, Foster C. Virtual support for paediatric HIV treatment decision making. Arch Dis Child 2015;100:527-31.

5. Shearer WT, Quinn TC, LaRussa P, et al. Viral load and disease progression in infants infected with human immunodeficiency virus type 1. Women and Infants Transmission Study Group. N Engl J Med 1997;336:1337-42.

6. Panel on Antiretroviral Therapy and Medical Management of HIV-1 Infected Children. Guidelines for the use of antiretroviral agents in pediatric HIV infection, 2013. (http://aidsinfo.nih.gov/contentfiles/lvguidelines/ pediatricguidelines.pdf.)

7. Van Dyke RB, Lee S, Johnson GM, et al.; Pediatric AIDS Clinical Trials Group Adherence Subcommittee Pediatric AIDS Clinical Trials Group 377 Study Team. Reported adherence as a determinant of response to highly active antiretroviral therapy in children who have human immunodeficiency virus infection. Pediatrics 2002;109:e61.

8. Blanche S, Bologna R, Cahn P, et al. Pharmacokinetics, safety and efficacy of darunavir/ritonavir in treatment-experienced children and adolescents. AIDS 2009;23:2005-13.

9. Neely M, Kovacs A. Managing treatment-experienced pediatric and adolescent HIV patients: role of darunavir. Ther Clin Risk Manag 2009;5: 595-615.

10. Wensing AM, Calvez V, Günthard HF, et al. 2014 Update of the drug resistance mutations in HIV-1. Top Antivir Med 2014;22:642-50.

11. Kirk BL, Gomila A, Matshaba M, et al. Early outcomes of darunavir- and/ or raltegravir-based antiretroviral therapy in children with multidrugresistant HIV at a pediatric center in Botswana. J Int Assoc Provid AIDS Care 2013;12:90-4. 
12. Thuret I, Chaix ML, Tamalet C, et al. Raltegravir, etravirine and r-darunavir combination in adolescents with multidrug-resistant virus. AIDS 2009;23:2364-6.

13. Rojas Sánchez P, de Mulder M, Fernandez-Cooke E, et al.; Madrid Cohort of HIV-Infected Children and Adolescents Integrated in the Paediatric Branch of the Spanish National AIDS Network (CoRISPe). Clinical and virologic follow-up in perinatally HIV-1-infected children and adolescents in Madrid with triple-class antiretroviral drug-resistant viruses. Clin Microbiol Infect 2015;21:605.e1-9.

14. Tudor-Williams G, Cahn P, Chokephaibulkit K, et al.; PIANO study group. Etravirine in treatment-experienced, HIV-1-infected children and adolescents: 48-week safety, efficacy and resistance analysis of the phase II PIANO study. HIV Med 2014;15:513-24.

15. Briz V, León-Leal JA, Palladino C, et al. Potent and sustained antiviral response of raltegravir-based highly active antiretroviral therapy in HIV type 1-infected children and adolescents. Pediatr Infect Dis J 2012;31: 273-7.

16. Leonard EG, McComsey GA. Metabolic complications of antiretroviral therapy in children. Pediatr Infect Dis J 2003;22:77-84.
17. Gupta RK, Gibb DM, Pillay D. Management of paediatric HIV-1 resistance. Curr Opin Infect Dis 2009;22:256-63.

18. Abrams EJ, Weedon J, Steketee RW, et al. Association of human immunodeficiency virus (HIV) load early in life with disease progression among HIV-infected infants. New York City Perinatal HIV Transmission Collaborative Study Group. J Infect Dis 1998;178:101-8.

19. Working Group on Antiretroviral Therapy and Medical Management of HIV-Infected Children. Guidelines for the use of antiretroviral agents in pediatric HIV infection. 23 February 2009, 2009. (http://aidsinfo.nih.gov/ ContentFiles/PediatricGuidelines.pdf.)

20. Durant J, Clevenbergh P, Halfon P, et al. Drug-resistance genotyping in HIV-1 therapy: the VIRADAPT randomised controlled trial. Lancet 1999;353:2195-9.

21. Clevenbergh P, Durant J, Halfon P, et al. Persisting long-term benefit of genotype-guided treatment for HIV-infected patients failing HAART. The Viradapt Study: week 48 follow-up. Antivir Ther 2000;5:65-70.

22. Green H, Gibb DM, Compagnucci A, et al.; Paediatric European Network for the Treatment of AIDS. A randomized controlled trial of genotypic HIV drug resistance testing in HIV-1-infected children: the PERA (PENTA 8) trial. Antivir Ther 2006;11:857-67. 